\title{
LA INDUSTRIA DEL CALZADO ESPAÑOLA EN LA POSGUERRA: LOS EFECTOS DEL INTERVENCIONISMO SOBRE UNA INDUSTRIA DE BIENES DE CONSUMO ${ }^{1}$
}

\author{
JOSE ANTONIO MIRANDA ENCARNACION \\ Universidad de Alicante
}

\section{RESUMEN}

Después de la guerra civil de 1936-39, la economia española atravesó un largo período de estancamiento. Entre las causas que lo originaron destaca una política económica de carácter intervencionista especialmente desafortunada. A pesar de que los dirigentes del nuevo régimen dieron prioridad a la industrialización del país, pocos sectores industriales consiguieron desarrollarse. Este articulo muestra cuáles fueron los efectos de la politica autárquica sobre la industria del calzado, una rama representativa del conjunto de las industrias ligeras, productoras de bienes de consumo. Se analiza la situación de la demanda, cómo evolucionó la producción y cómo lo hicieron también la dotación tecnológica y la productividad. Finalmente, se estudia cuál fue la actitud empresarial ante el intervencionismo del Estado.

\section{ABSTRACT}

The policy of strict regulation followed by the state, accounts for the unsuccessful performance of the Spanish economy after the 1936-39 Civil War. Despite the emphasis on industrial growth of the new political regime, few manufacturing sectors attained a successful performance. This paper shows the effects of the Spanish autarchic policy on the shoe-making industry, a representative branch of consumer goods industries. Demand, production, technology and entrepreneurial attitudes are analyzed in connection with the intervention of the state.

\section{INTRODUCCION}

Los ya abundantes trabajos sobre la economía española durante el primer franquismo han caracterizado el periodo, y especialmente la década de 1940 , como una época de estancamiento cuando no de auténtico retroceso económi-

1 Este trabajo es una parte de la tesis doctoral que realizo con una beca del Banco de España. 
co. Algunos indicadores a este respecto son claros: en la agricultura, un cultivo tan esencial como el trigo veía disminuir su producción en una cuarta parte respecto a los niveles de preguerra ${ }^{2}$; en la industria, mientras el producto español tenía una tasa de crecimiento anual del 0,58 por ciento entre 1935 y 1950 , en el conjunto de Europa el crecimiento superaba el 2,7 por ciento ${ }^{3}$, y del descenso del nivel de vida es buena muestra la reducción a la mitad del poder adquisitivo de los trabajadores en ese mismo periodo ${ }^{4}$. Un negro panorama que interrumpió el moderado crecimiento que se sostenia desde el último tercio del siglo XIX y que alejó aún más a nuestro país de las cotas alcanzadas por sus vecinos europeos 5 .

Entre las causas del retroceso se ha señalado la importancia de una política económica especialmente desafortunada. El nuevo régimen surgido tras la guerra civil radicalizó las tendencias aislacionistas e intervencionistas que apuntaban en la política económica española desde comienzos del siglo $\mathrm{xx}^{6}$, aplicando una politica de intervenciones y controles que aspiraba a la independencia económica del pais, a la autarquía.

Durante la primera mitad de la década de 1940, esta politica incrementó los estrangulamientos (en materias primas, tecnologia y productos energéticos) originados por la Segunda Guerra Mundial y mermó el provecho que España, como estado neutral, podía haber obtenido de la coyuntura bélica ${ }^{7}$. En la segunda parte de la década, el nacionalismo económico contribuyó a retrasar el proceso de recuperación y condicionó el crecimiento que vendría en los años cincuenta ${ }^{8}$.

A pesar de que los dirigentes del régimen dieron prioridad a la industrialización del país ${ }^{9}$, el fracaso de la política industrial fue rotundo. Las empresas padecieron escasez de materias primas y energia, y la represión política afectó con fuerza a su capital humano ${ }^{10}$. También se tuvo que sufrir la caida del consumo privado "', la insuficiente red de transportes y las dificultades para modernizar el aparato productivo ${ }^{12}$. En algunos sectores, el daño se vio incrementado por un sistema de cupos y tasas fijado con criterios extraeconómi-

\footnotetext{
2 Barciela (1987).

3 Carreras (1990), p. 83.

4 Molinero e Ysas (1985), Carreras (1989).

s Garcia Delgado (1987).

6 Clavera, Esteban, Mones, Montserrat y Ros (1973).

7 Catalán (1989).

8 Catalan (1992).

* Gonzälez (1979).

10 Catalán (1989).

11 Carreras (1985).

12 Catalan (1991).
} 
$\cos { }^{13}$, el establecimiento de barreras legales para la entrada en la industria ${ }^{14}$ y la generalización de prácticas fraudulentas ${ }^{15}$.

La autarquía perjudicó en mayor medida a las industrias de bienes de consumo ${ }^{16}$. En las páginas que siguen vamos a observar cuáles fueron sus efectos sobre el sector del calzado y repasaremos la situación del consumo de este producto durante los años cuarenta, cómo evolucionó su producción y cómo lo hicieron también la dotación tecnológica y la productividad.

\section{LA HERENCIA DE LA GUERRA CIVIL}

La guerra civil no ocasionó grandes destrucciones en las empresas de calzado, como tampoco las ocasionó en el conjunto de la industria ${ }^{17}$. Tan sólo alli donde se estabilizaron los frentes hubo daños importantes; fue el caso de una de las empresas que mayores dimensiones había alcanzado y sobre la que volveremos más adelante, la de «Silvestre Segarra e Hijos», en Vall de Uxó, Castellón, que fue desmantelada y su maquinaria repartida por distintas provincias ${ }^{18}$. En el resto del país apenas hubo pérdidas materiales y las incautaciones y controles obreros tampoco alteraron en exceso un equipo productivo que se mantenia activo para abastecer la demanda militar ${ }^{19}$. Gracias a esta demanda se consiguieron incluso buenos beneficios en muchas empresas: en Elche, uno de los principales municipios zapateros del país, el Consejo de Economia Local, que se encargaba de la comercialización del producto, obtuvo unas ganancias de 1.183 .123 pesetas en el año 1937 y de 1.835 .968 en 1938; y la empresa de calzado textil «Ferrández y Garcia» multiplicó en el año 1937 por 2,5 los beneficios declarados en $1935^{20}$. En el otro gran centro del calzado alicantino, Elda, la empresa «Rodolfo Guarinos» declaró entre julio y diciembre de 1936 más de 400.000 pesetas de beneficios y en los tres primeros

13 Un estudio ejemplar de las repercusiones de este sistema, aunque aplicado a la producción agraria, es el de Carlos Barciela (1985).

14 Buesa (1984).

15 Garcia Delgado (1986).

I6 Sudrià (1991), Molinero (1991).

17 Clavera (1976), Malefakis (1987), pp. 162-163; Calvet (1992), pp. 48-55.

18 Gaja y Ferrer (1964).

19 En las declaraciones efectuadas por los empresarios para instruir la Causa General (Archivo Histórico Nacional), raramente se mencionan daños materiales y como perjuicios sufridos se limitan a destacar la disminución del capital circulante.

20 Véase la declaración del impuesto de utilidades de la sociedad «Ferrández y Garcia». AHPA, HG, leg. 715 . 
meses de 1937 éstos sobrepasaron el millón ${ }^{21}$. En Villena, las principales empresas, agrupadas en «Fabricalzado, I.S.», consiguieron entre febrero y diciembre de 1937 más de 300.000 pesetas de beneficio 22 .

La capacidad productiva no era menor en 1939 que en 1936; al contrario: como la mayor parte de la industria del calzado estaba situada en el territorio que quedó bajo el control de la República, el ejército insurrecto tuvo que fomentar la producción en aquellas zonas que dominaba para conseguir abastecerse de botas, zapatos y alpargatas. El resultado fue que en 1938 la producción de calzado en la España nacional era un 53 por ciento mayor que en $1936^{23}$. Este crecimiento estuvo capitalizado por la industria mallorquina, que, de manera semejante a lo sucedido en Béjar con la industria textil lanera ${ }^{24}$, se vio favorecida por la falta de competencia y duplicó su producción durante la guerra.

Mientras Menorca se alineaba con la España republicana, Mallorca estuvo con los nacionales desde 1936; el aislamiento inicial que le ocasionó su posición paralizó la industria por la falta de materiales y las dificultades de comunicación ${ }^{25}$, pero pronto se consiguió regularizar el tráfico marítimo $\mathrm{y}$, a pesar de la escasez de cueros, la isla llegó a fabricar 2,9 millones de pares de botas para el ejército durante los años de la guerra civil, y el número de fábricas pasó de 105 en 1937 a 250 en 1939 , y el de empleados de 4.267 a $7.500{ }^{26}$.

Para las nuevas empresas surgidas en la zona nacional, el final de la guerra significó más severas restricciones de materias primas y tener que comenzar a competir con la industria del Mediterráneo español. Las empresas del territorio recién conquistado tuvieron que hacer frente, en primer lugar, a la carencia de circulante, ocasionada por el bloqueo de los saldos, la inmovilización de los créditos gestionados durante la guerra y las dificultades para reactivar los créditos anteriores a julio de 1936. A ello se añadía la necesidad de reponer o reparar maquinaria y rehacer las plantillas de trabajadores y personal técnico y directivo. No se disponía de materias primas en cantidad suficiente, tanto por la caida de las importaciones como por la escasa producción nacional y su defectuosa distribución. Algunas empresas, además, tuvieron que sufrir una «in-

21 Santacreu (1992).

22 Véanse los datos complementarios al movimiento de capital del Comité Local de Control y Socialización del Ramo de la Piel y Calzado. AHN.SGC, PS-Alicante, legs. 56/15.

23 Malefakis (1987), pp. 162.

24 Calvet (1992), pp. 45-47.

25 Gayoso (1991), pp. 168.

26 Presidencia del Gobierno-INE (1951), p. 316. 


\section{GRAFICO 1}

Industria mallorquina del calzado, 1937-1945

Producción y número de empresas

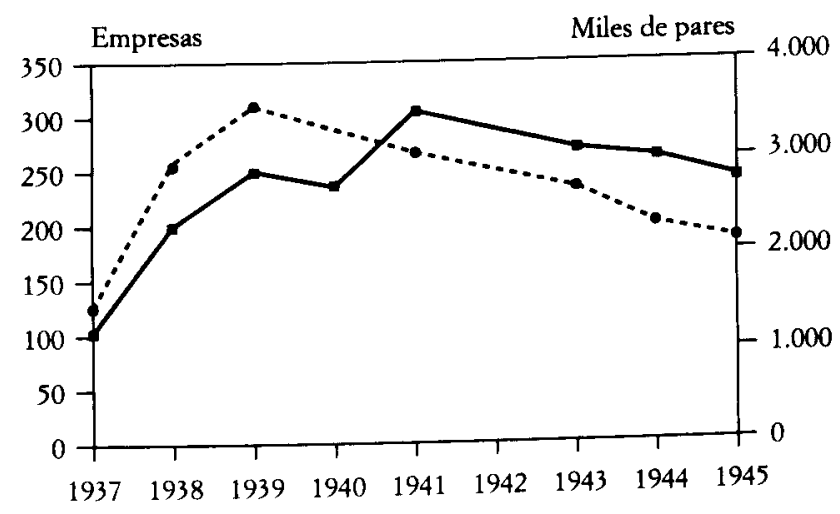

Fuente: Piel. Revista española de las industrias de la piel, núm. 104, 1952, pp. 23-25.

terpretación demasiado extensiva del concepto botín de guerran, sobre sus depósitos de materias primas y manufacturas ${ }^{27}$.

En general, la industria española del calzado de piel abandonó la década de 1930 con una estructura de más de 1.500 empresas y 40.000 trabajadores, con cerca de un millar de máquinas de montar; también se contaba con 2.300 talleres de calzado a medida y más de 8.000 operarios en ellos ${ }^{28}$. Su capacidad de producción era de unos 32 millones de pares anuales.

Este aparato productivo tenía su mayor concentración en la provincia de Alicante, con 334 empresas, casi 14.000 operarios y el mayor número de maquinaria de montaje, sobre todo por el sistema mixto. La seguian Baleares y Barcelona. Las islas, con 340 empresas de reducido tamaño y 9.000 trabajadores, se decantaban por el montaje Goodyear. Barcelona tenía una industria zapatera mucho menos importante, que había ido retrocediendo en relación a las anteriores provincias desde principios de siglo y que entonces constaba de 160 empresas y 3.500 trabajadores. Del resto de las provincias, Zaragoza tenía un buen número de empresas, operarios y maquinaria, al igual que Valencia y

${ }^{27}$ Véase el informe confidencial sobre la situación industrial en Cataluña de 1940. AGA, Sección de la Presidencia del Gobierno, Caja 30.

28 Carrió (1945), p. 19. 
Logroño. Albacete, con su industria concentrada en Almansa, y Castellón, con igual fenómeno en Vall de Uxó, disponían de empresas grandes, muy mecanizadas. Huelva se caracterizaba por su nube de pequeños talleres manuales y por el resto de España se repartían fábricas y talleres con una importancia meramente local o provincial en el mejor de los casos.

Aunque la especialización de determinadas localidades en la industria del calzado era una realidad evidente desde principios de siglo, la guerra civil y las dos primeras décadas del franquismo frenaron el proceso de concentración geográfica. Durante la guerra, la división del país en zonas rivales alentó el desarrollo de empresas de calzado en otras provincias; en la posguerra, estas empresas se mantuvieron gracias al sistema de intervención y se vieron favorecidas por la debilidad del mercado nacional: las dificultades en los transportes, sobre todo para las materias primas, y la caída de la capacidad adquisitiva de la población generaron en las provincias una tendencia al autoabastecimiento. Se dio incluso una fuga de empresas desde los distritos industriales del calzado hacia el resto de provincias ${ }^{29}$.

\section{GRAFICO 2}

Producción de calzado por provincias. Promedio anual 1933-1935

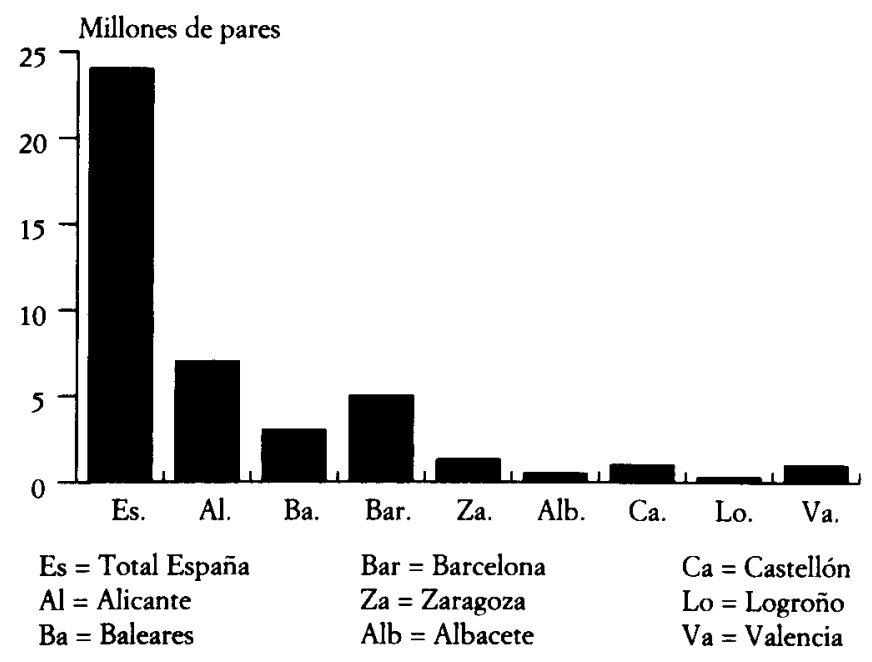

${ }^{29}$ AGA, Sección del Ministerio de Industria, Cajas 5.391 y 6.146. 
El calzado de cuero se veía acompañado por un subsector de calzado textil que comprendía más de un millar de empresas y cerca de 20.000 operarios. La mayor parte de la producción se realizaba en la zona de Levante y especialmente en la provincia de Alicante, que debía reunir en torno al 60 por ciento de la industria alpargatera española y que tenia en el municipio de Elche su principal centro de fabricación.

Hasta los años veinte, la alpargata fue un producto de amplísimo consumo. A mediados de la década, sin embargo, el incremento del nivel de vida se dejó notar en una mayor demanda para el calzado de piel y la alpargata tradicional, con suela de fibra, fue progresivamente sustituida por la de suela de goma, a la vez que se difundian otros tipos intermedios de calzado, como las zapatillas de piel ${ }^{30}$. Este proceso se vio bruscamente interrumpido por la guerra civil, que convirtió el zapato en un producto suntuario y volcó la demanda masiva hacia la alpargata, recuperando el uso de las fibras textiles por la escasez y mala calidad de la goma disponible.

\section{EL ESTANCAMIENTO DE LA PRODUCCION: LA RESPONSABILIDAD DE LA DEMANDA}

Aunque contaba con un aparato productivo mayor, la producción real de calzado disminuyó respecto a los niveles de preguerra. Si el trienio 1933-1935 tuvo una producción media de 24 millones de pares de calzado de piel, esta cantidad no volvió a alcanzarse hasta bien entrada la década de 1950. La escasez de materias primas y el hundimiento de la capacidad de consumo de la población española, principalmente, pero también las dificultades para la exportación y las restricciones eléctricas, mantuvieron a la industria con una producción casi estancada, un 40 por ciento por debajo de su capacidad de oferta.

El retroceso fue similar en su tamaño al experimentado por el conjunto de la industria española de bienes de consumo manufacturados, pero no en su ritmo, más acentuado en los primeros años para las otras industrias, mientras que para el calzado la caida se agravó hacia mediados de la década.

Durante los primeros años de posguerra, los pedidos oficiales y la demanda civil insatisfecha que se había acumulado a lo largo del conflicto mantuvieron un consumo interior de calzado relativamente alto, por lo que también se

30 Miranda (1991). 


\section{CUADRO 1}

Producción española de bienes de consumo manufacturados en general y de calzado Indice 1935-100

\begin{tabular}{|c|c|c|}
\hline Año & Industrias de bienes de consumo & Industria del calzado \\
\hline $1935 .$. & 100 & 100 \\
\hline 1940. & 69 & 80 \\
\hline 1945. & 81 & 70 \\
\hline $1950 .$. & 80 & 81 \\
\hline
\end{tabular}

Fuentes: Carreras (1983) —-tomamos los datos a través de Sudrià (1991) - y Servicio Sindical de Estadística.

mantuvo la producción y, a pesar de la legislación restrictiva sobre nuevas industrias ${ }^{31}$, continuaron estableciéndose empresas. Los precios estaban tasados y apenas se disponia de materiales, pero el recurso a sustitutivos y el fraude en la calidad del producto permitian obtener buenas ganancias.

Esta situación varió a partir de 1943, entrando el consumo interior en una atonía de la que no se recuperaría hasta la década siguiente, sobre todo en el caso del calzado de piel. Mientras en 1936 se consumian anualmente 70 pares de zapatos por cada 100 habitantes, doce años después esta cifra se habia reducido a casi la mitad ${ }^{32}$, pues la mayoría de la población calzaba alpargatas o zapatillas. El valor de las ventas de pares de cuero en pesetas constantes pasó de un indice 100 en 1943 a otro 69,2 en 1946.

Existia un grave problema de demanda por la caida del poder adquisitivo de la mayoria de la población, que se veía obligada a recurrir a calzados alternativos (alpargatas, zapatillas, calzado vulcanizado), y por el cese de las exportaciones. En el quinquenio 1931-1935 las ventas de calzado de piel a otros paises (Francia y sus posesiones norteafricanas, las Antillas y Alemania) superaron las 85 toneladas anuales y las de alpargatas (a los lugares ya citados y, en menor proporción, a Oriente Medio) los 2,5 millones de pares. Esta corriente se

31 Miranda y Pérez Ortiz (1992).

32 Las regiones con un mayor consumo de calzado de cuero, en relación al número de habitantes, eran Asturias y el País Vasco, con un 65 por ciento de su población que utilizaba este calzado, y Cataluña, Baleares y Navarra, con un 50 . Entre las regiones con un consumo más bajo destacaban Andalucía y Extremadura, con apenas un 20 por ciento (Piel. Revista española de las industrias de la piel, abril de 1948, p. 15). 


\section{GRAFICO 3a}

Producción de calzado en España, 1940-1952

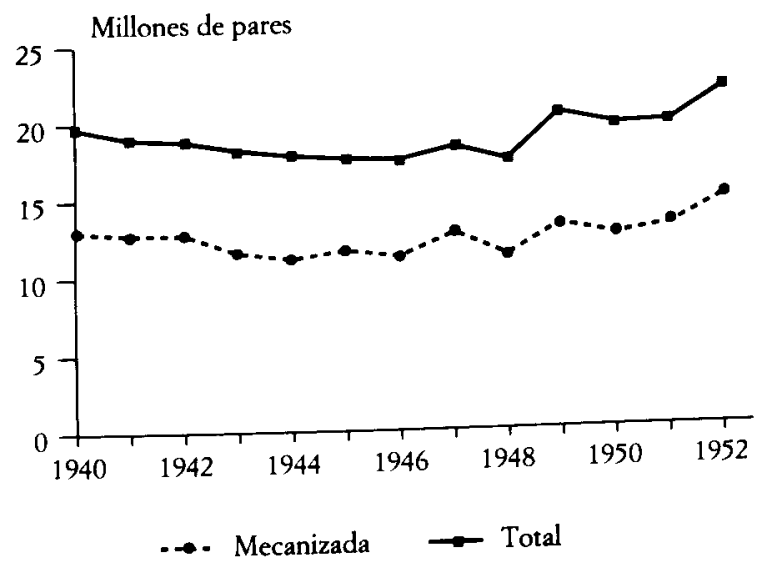

FUENTE: Servicio Sindical de Estadistica.

\section{GRAFICO 3b}

Principales provincias productoras de calzado, 1945-1951

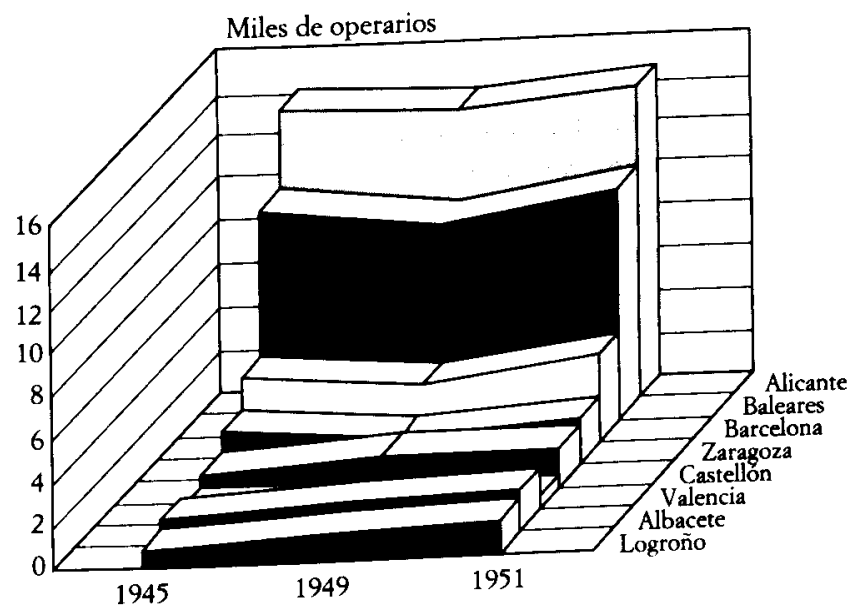


interrumpió casi por completo con la guerra civil y, aunque comenzó a recuperarse en la segunda mitad de los años cuarenta, no alcanzaría un valor importante hasta la década siguiente.

La sobrevaloración del tipo de cambio de la peseta dificultaba la entrada en los mercados internacionales, pero tampoco las empresas españolas estaban en condiciones de fabricar un calzado competitivo. Por ello, las medidas de fomento de las exportaciones aplicadas en 1946 (sistemas de admisiones temporales e importaciones con exención de derechos arancelarios) y en 1948 (cambios variables para cada producto) apenas se dejaron sentir ${ }^{33}$.

\section{INTERVENCION, MATERIAS PRIMAS Y MERCADO NEGRO}

Más que producir competitivamente, el reto era lograr producir. Ante todo, por la escasez de materiales. Los cueros vacunos disponibles pasaron de una media anual de 38.000 toneladas (peso en sangre) en el período $1931-1935$ a una media de 24.000 entre 1940 y 1944 , y a menos de 18.000 toneladas en el quinquenio siguiente. La clave del problema residía en la intervención estatal, que afectó tanto a las importaciones como a la producción interior de cueros.

\section{a) La caída de las importaciones}

Aunque con una buena producción de pieles lanares y cabrías, España siempre había sido deficitaria en cueros de vacuno y equino, para cuyo abastecimiento dependía del comercio exterior. En consecuencia, la principal causa de la reducción de los cueros disponibles fue la caida de las importaciones; éstas pasaron de una media de 7,5 toneladas de cueros vacunos en términos de cuero seco durante los primeros años de la década de 1930 a tan sólo 2 toneladas en los años cuarenta.

Hasta 1935 las importaciones españolas de cueros se habían realizado en completa libertad. A partir de aquel año se estableció un contingente que hizo disminuir las compras y con el establecimiento del régimen de Franco se impuso una absoluta intervención sobre la importación de cueros y pieles, en

${ }^{33}$ Orden del Ministerio de Industria y Comercio del 3 de diciembre de 1948 (BOE del 12 de diciembre). 
manos primero del Sindicato Vertical de la Piel y, desde 1948, de la Comisaría General de Abastecimientos y Transportes ${ }^{34}$.

La coyuntura internacional dificultaba las importaciones, ante la escasez de fletes y el alto consumo de cueros por parte de los países en guerra, pero las circunstancias políticas y las directrices económicas españolas las frenaron en mayor medida y buena prueba de ello es que el hundimiento de las compras exteriores de cueros fue mayor en la segunda mitad de la década de 1940 que durante los años de la Guerra Mundial. Las pretensiones autárquicas del régimen y, particularmente, la escasez de divisas, consecuencia de la guerra civil y de la nefasta estrategia económica desarrollada tras ella ${ }^{35}$, redujo las importaciones de cueros a la mitad de las necesarias a lo largo de toda la década; incluso en 1948, cuando la firma del Protocolo Franco-Perón consiguió aliviar la dramática escasez de los tres años anteriores, el problema siguió siendo grave.

\section{CUADRO 2}

Procedencia del cuero vacuno importado en España, 1932-1942 (porcentajes)

\begin{tabular}{|c|c|c|}
\hline País & $1932-34$ & $1940-42$ \\
\hline Argentina $\ldots \ldots \ldots \ldots \ldots \ldots \ldots \ldots$ & 38,9 & 66,4 \\
\hline Gran Bretaña y colonias ..................... & 20,7 & 28,4 \\
\hline Francia y colonias .......... & 19 & 0,3 \\
\hline Holanda y colonias ........ & 9,9 & 0,8 \\
\hline 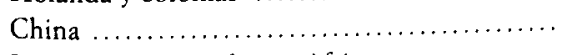 & 3,2 & 0 \\
\hline Posesiones españolas en Africa .............. & 1 & 0 \\
\hline Otros paises americanos $\ldots \ldots \ldots \ldots \ldots \ldots \ldots$ & 3 & 0,07 \\
\hline 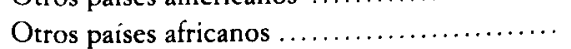 & 0,01 & 3,7 \\
\hline
\end{tabular}

FuenTE: Servicio Sindical de Estadistica.

Tradicionalmente Argentina habia sido el principal abastecedor, proporcionando más de una tercera parte de los cueros foráneos consumidos en España durante la primera mitad de los años treinta. Otros suministradores importantes eran Reino Unido y sus colonias y Francia, también con su imperio colonial. Pero cuando comenzó la Segunda Guerra Mundial los suministros ar-

34 Orden ministerial del 31 de diciembre de 1940 (BOE del 1 de enero de 1941).

35 Catalán (1991). 
gentinos se convirtieron en esenciales, representando un 66 por ciento del total de cueros importados; entonces la colonia inglesa de Nigeria pasó a ser la segunda gran fuente de cueros para España, con cerca del 30 por ciento.

\section{b) La intervención sobre los cueros del pais: el mercado negro}

La debilidad de las importaciones estaba agravada por la errónea politica de control de la producción interior de cueros, que hizo que éstos disminuyesen y se desviasen en gran parte hacia el mercado negro. A lo largo de la década de 1940 la recogida oficial de cueros vacunos se mantuvo en una media anual inferior al millón de unidades y si no tenemos en cuenta las cifras de 1944 y 1949, años con una producción extraordinariamente alta, esta media se reduce a 875.000 cueros por año. Las principales zonas de recogida eran las grandes ciudades, sobre todo Madrid y Barcelona, por el alto número de reses sacrificadas en sus mataderos, y las tradicionales regiones ganaderas de la España húmeda: Galicia, Asturias, Cantabria y el País Vasco.

Los cueros se intervenían en origen, en los mismos mataderos o conforme se realizaban las importaciones, y se asignaban mediante cupos a los curtidores $y$, el producto de éstos, a las empresas de calzado y otras manufacturas. De esta manera, las empresas carecian de alicientes para mejorar su producción, ya que dependian de asignaciones fijas (en función de una teórica capacidad productiva que no se podia modificar) de materias primas escasas y sus precios estaban tasados, con lo que la mejor manera de obtener beneficios era envileciendo la calidad del producto.

El sistema fue sustituido en 1944 por el de "cupos de reposición», que introducía cierta libertad en la industria. Se mantenian los cupos para las empresas que manufacturaban la piel, pero se permitia a éstas comprar las materias primas eligiendo entre los distintos distribuidores. Los cueros sin curtir se repartían en función de los pedidos acumulados por cada empresa curtidora. Con este nuevo sistema se pretendía estimular la competitividad y el rendimiento de las tenerías, mejorando también la calidad. Pero nada se hizo por remediar los mismos males en las empresas de calzado y, de cualquier forma, la medida no podía ser efectiva porque seguía existiendo un cuello de botella en el abastecimiento de cueros y productos curtientes.

Otro problema añadido era la magnitud del comercio clandestino. En 1943 los mismos responsables administrativos de la intervención calculaban que el déficit de cueros vacunos para la industria era de 17.000 toneladas (en 
términos de peso en sangre), que se habían controlado entre un 15 y un 20 por ciento menos de los cueros realmente obtenidos en el país y que la producción de curtidos intervenida era la mitad de la normal en los años treinta.

El volumen del mercado negro dependia en buena parte de los suministros exteriores: cuando disminuia la importación, los cupos oficiales también lo hacian y las empresas necesitaban comprar mayores cantidades en el mercado negro. A su vez, esta mayor demanda de comercio ilícito incrementaba los precios pagados en él y, en consecuencia, los estímulos para sustraer cueros a la recogida oficial. Según cálculos del Sindicato Vertical, en 1945 los repartos oficiales de suela y piel de empeine sólo hubieran permitido elaborar 4 millones de pares de calzado civil y la producción real fue más de tres veces superior.

El recurso al mercado negro también era necesario para proveerse del resto de materias primas y accesorios (clavazón, alambre para cosido, colas...) que requería el calzado. Estos materiales se encontraban intervenidos y dependían de distintos Sindicatos (del metal, de industrias quimicas...). Al Sindicato Vertical de la Piel se le concedían unos cupos globales que éste se encargaba de distribuir entre las empresas. Las necesidades de clavazón, por ejemplo, que se calculaban en 1,3 toneladas, sólo fueron cubiertas oficialmente en el año 1945 en un 10 por ciento.

\section{GRAFICO 4}

Cueros vacunos disponibles para la industria española, 1926-1952

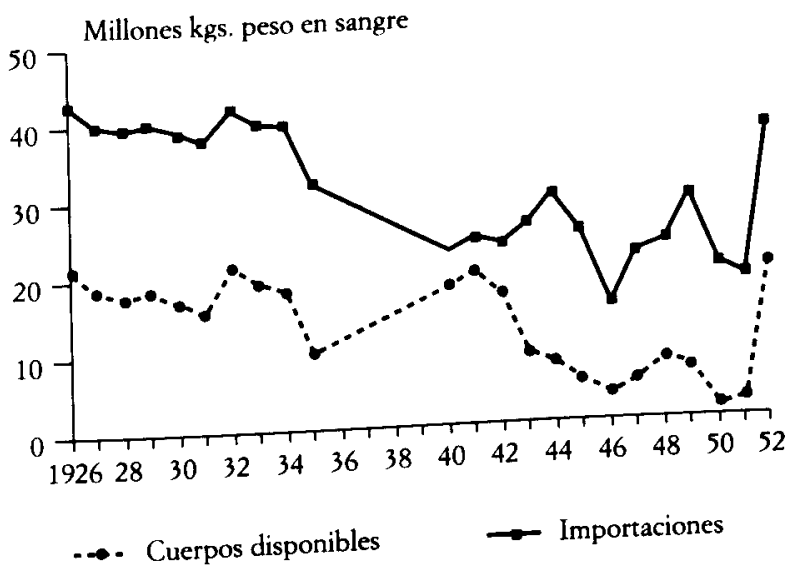

FuENTE: Servicio Sindical de Estadística. 
La falta de materias primas se agravaba por otra decisión política: la prioridad dada a los suministros para el ejército y los cuerpos de orden público y el volumen alcanzado por éstos. Si en 1935 los suministros oficiales sumaron 150.000 pares, nueve años más tarde superaban los 3 millones, de modo que entre el 50 y el 80 por ciento de la suela y el empeine producidos con cueros del pais estuvo destinado en la década de 1940 a la demanda institucional.

Los suministros oficiales se hacian con bastante independencia de la mayor o menor disponibilidad de cueros, pues la asignación de materiales para ellos era la primera tarea que se realizaba una vez conocidas, cada mes, la producción y las importaciones de esta materia prima, y eran los usos civiles del cuero, a los que se destinaba el resto, los que tenían que sufrir la escasez. Por ello, entre otras medidas encaminadas a mejorar la situación de su industria, los empresarios de Mallorca solicitaban a mediados de la década una «justa distribución de la materia prima, en lo que hace referencia a los suministros oficiales» o, en su defecto, «un sistema de compensación a favor de la industria que solamente puede destinar su producción al consumo civil» ${ }^{36}$.

\section{CUADRO 3}

Recogida de cueros en España por el Sindicato Nacional de la Piel, 1943

\begin{tabular}{|c|c|c|}
\hline Tipo de cuero & $\begin{array}{l}\text { Kgs. (en fresco) } \\
\text { recogidos }\end{array}$ & $\begin{array}{c}\text { Porcentaje } \\
\text { para el calzado civil }\end{array}$ \\
\hline Para empeine (box calf, cueros hasta $8 \mathrm{kgs}$.) ... & 263.070 & 20 \\
\hline $\begin{array}{c}\text { Para empeine (becerro y box calf, cueros } \\
\text { tipo } 8 / 18, \ldots \ldots \ldots \ldots \ldots \ldots \ldots \ldots \ldots \ldots \ldots \ldots \ldots\end{array}$ & 3.791 .298 & 40 \\
\hline Para suela (cueros tipo $18 / 30) \ldots \ldots \ldots \ldots \ldots$ & 5.449 .466 & 18 \\
\hline Para suela (cueros tipo $30 / 40$ ) & 2.543 .739 & 12,5 \\
\hline
\end{tabular}

Fuente: Servicio Sindical de Estadística.

Los pedidos de las fuerzas armadas se adjudicaban mediante subasta y solian recaer en las empresas más fuertes y mejor relacionadas con la Administración. En las concesiones que se hicieron en julio de 1945, por ejemplo, la empresa «Silvestre Segarra e Hijos», una de las más apoyadas desde el poder, consiguió una tercera parte de los suministros para el Ejército de Tierra y más de la mitad de los solicitados por las Fuerzas Aéreas. El mismo Sindicato de la

36 COCIN de Palma de Mallorca (1946), p. 128. 
Piel se quejaba, en el III Consejo Sindical Industrial, de la existencia de «fabricantes privilegiados» en la adjudicación de pedidos militares.

Ante la escasez de materiales, la respuesta de las empresas fue recurrir a sustitutivos. Las pieles lanares y cabrías se utilizaron como sucedáneos de los cueros vacunos; para elaborar punteras y contrafuertes se recurrió a «tela de La Haye» (fibras textiles con un engomado soluble al agua), al cartón y a fieltros cromados; para las sandalias se emplearon plantillas de madera y, en otros tipos de calzado, los pisos a base de caucho y regenerados de este material, cáñamo, yute y esparto ${ }^{37}$.

Pero incluso estos materiales eran escasos. Las importaciones de caucho sin labrar y en objetos inutilizados pasaron de 247.000 quintales en 1935 a 36.000 en 1940; otro tanto sucedió con el yute; la lona de algodón no era fácil de conseguir en los primeros años de posguerra y el cáñamo alcanzó el prohibitivo precio de 1.000 pesetas el quintal métrico ${ }^{38}$. La escasez era tal que en 1943 se llegó a prohibir la venta de calzado con piso de goma si el comprador no entregaba el par usado ${ }^{39}$. La alternativa al calzado de cuero, por tanto, eran unas alpargatas y zapatillas de calidad ínfima, porque las suelas de goma se hacian con regenerados procedentes de anteriores procesos de regeneración, hasta que perdían toda flexibilidad, y las de suela de fibra lo eran de esparto encapado superficialmente con cáñamo, sin resistencia ninguna ${ }^{40}$.

\section{LAS DIFICULTADES CON LA ENERGIA}

La producción también se vio frenada por la pobre oferta de energía, especialmente de electricidad. El crecimiento del consumo eléctrico en nuestro país no habia ido seguido de un paralelo aumento de la capacidad productiva de las compañias suministradoras, entre otras razones porque las tarifas estaban congeladas y se había desanimado la inversión en el sector ${ }^{41}$. El resultado fue que desde principios de los años cuarenta se soportaron severas restricciones. Los recortes en el suministro fueron acompañados de una menor calidad del flujo, con alteraciones en la frecuencia y el voltaje ${ }^{42}$, y se dejaron

37 Una descripción completa de como se conseguia un calzado minimamente resistente con materiales malos y escasos puede verse en la solicitud de R. Pérez para ampliar su fábrica de calzado en 1943. AGA, Sección del Ministerio de Industria, Caja 5.509.

38 AGA, Sección del Ministerio de Industria, Caja 5.493.

39 Orden del 10 de marzo de 1943 ( $B O E$ de 19 de marzo)

4(1) AGA, Sección del Ministerio de Industria, Caja 5.422

41 Sudrià (1987).

42 AGA, Sección del Ministerio de Industria, Caja 7.164. 
sentir más en las industrias que no se encontraban entre las prioridades del gobierno, como era el caso del calzado.

Aunque el grueso de las restricciones comenzó en 1943, en algunos centros zapateros, como Mallorca, el problema se presentó ya en 1940. En la isla el consumo había crecido rápidamente y se tenia que abastecer con una anticuada central térmica a la que se asignaban unos cupos de carbón insuficientes ${ }^{43}$. Las empresas se vieron obligadas a instalar grupos electrógenos supletorios y, aunque la situación mejoró al cabo de unos años, con la llegada a la isla de una central móvil aportada por el Instituto Nacional de Industria, todavia en 1953 se padecía una restricción de fluido equivalente al suministro de tres días por semana ${ }^{44}$.

El consumo de energia eléctrica en Alicante, provincia que concentraba la mayor parte de la industria del calzado, estuvo en 1943 seis puntos por debajo del registrado en 1942; en 1944 esta diferencia fue de 29 puntos y en 1945 de $41{ }^{45}$. Se recurrió a los motores de carbón, gasolina, gas-oil y fuel-oil, pero era una inversión cara y poco rentable, ya que estos carburantes también se encontraban racionados. Aun asi, en 1944 fueron 26 las empresas de calzado que recibieron cupos de combustible en esta provincia para el funcionamiento de sus centrales térmicas particulares ${ }^{46}$.

La distribución de los carburantes se realizó también de acuerdo con las prioridades establecidas por las autoridades económicas. Ello perjudicó a la industria del calzado en general y favoreció a determinadas empresas, bien porque abastecían la demanda militar, bien por tratarse de empresas potentes, con unas buenas relaciones con la Administración. Así, a finales del año 1944, mientras que la mayoria de fábricas zapateras que habian solicitado cupos de combustibles líquidos recibía tan sólo una parte de las cantidades demandadas ${ }^{47}$, otras, como la de Venancio Riera, en Cocentaina, Alicante, conseguian su cupo completo, en este caso 8.000 litros de gas-oil al mes.

La discriminación también se efectuaba directamente en los repartos de electricidad, aunque de forma más disimulada. La empresa «Silvestre Segarra e

${ }^{43}$ Véase el expediente sobre restricciones eléctricas en Mallorca. AGA, Sección del Ministerio de Industria, Caja 6.995.

14 En Cataluña, todavia en agosto de 1954, los cortes de suministro se realizaban dos dias a la semana, desde las 7 a las 19 horas, habia turnos establecidos para la utilización de la energia y la industria del calzado tenia limitado su consumo de electricidad para fuerza motriz a un 60 por ciento del consumo normal (AGA, Sección del Ministerio de Industria, caja 7.246).

${ }^{45}$ Moreno (1988).

th AGA, Sección del Ministerio de Industria, Caja 7.164.

47 En torno a una tercera parte de lo solicitado (AGA, Sección del Ministerio de Industria, Caja 7.164). 
Hijos», por ejemplo, tenía que padecer en el verano de 1944 un corte de fluido total durante tres días a la semana, aparentemente como el resto de empresas del sector en la región valenciana, pero sólo aparentemente, pues en realidad se veía compensada por el suministro de electricidad los domingos y durante más horas a lo largo de la semana, hasta completar su producción normal ${ }^{48}$.

\section{LOS EFECTOS DE LA INTERVENCION SOBRE EL APARATO PRODUCTIVO}

No sólo se estancó la producción. El sistema de cupos y la protección del mercado interior desanimaron la búsqueda de una mayor productividad. Esta falta de estímulos para la mejora de las empresas e incluso su prohibición, mediante las leyes sobre instalación de nuevas industrias y modificación de las ya existentes, impidieron que el sector se modernizase. La maquinaria era costosa y difícil de conseguir, por lo que las empresas optaron por sustituir la inversión por el mantenimiento de una mano de obra que era barata y dócil. Este fenómeno afectó también a las empresas suministradoras de productos intermedios, hasta el punto de resucitar la elaboración manual en actividades, como la confección de trenza para alpargatas, que se habian mecanizado en el siglo XIX ${ }^{49}$.

En toda una década, después de tres años de guerra en los que no se importó maquinaria para el calzado, el número de aparatos de montar se incrementó en tan sólo once unidades y el resto del utillaje técnico corrió una suerte parecida. De las máquinas de montaje, casi la mitad utilizaba el sistema mixto, un 15 por ciento el sistema Goodyear y el resto montaba punteras-taloneras y sandalias. La productividad teórica media de estos ingenios era de 125 pares diarios, pero en realidad, debido a la falta de accesorios y por utilizarse también para la producción de zapatillas, su rendimiento no superaba los 100 pares.

La producción española de maquinaria para calzado era corta y se limitaba a la construcción de aparatos pequeños, de mecanismo sencillo, y de algunas piezas de recambio o accesorios para la maquinaria importada. También se

48 AGA, Sección del Ministerio de Industria, Caja 7.164.

49 AGA, Sección del Ministerio de Industria, Cajas 5.503 y 5.536. 
realizaban en el país las partes más pesadas de las máquinas de importación, con el fin de ahorrar divisas y hacer más fáciles y económicos los fletes ${ }^{50}$.

\section{GRAFICO 5}

Maquinaria en la industria del calzado española, 1944-1952

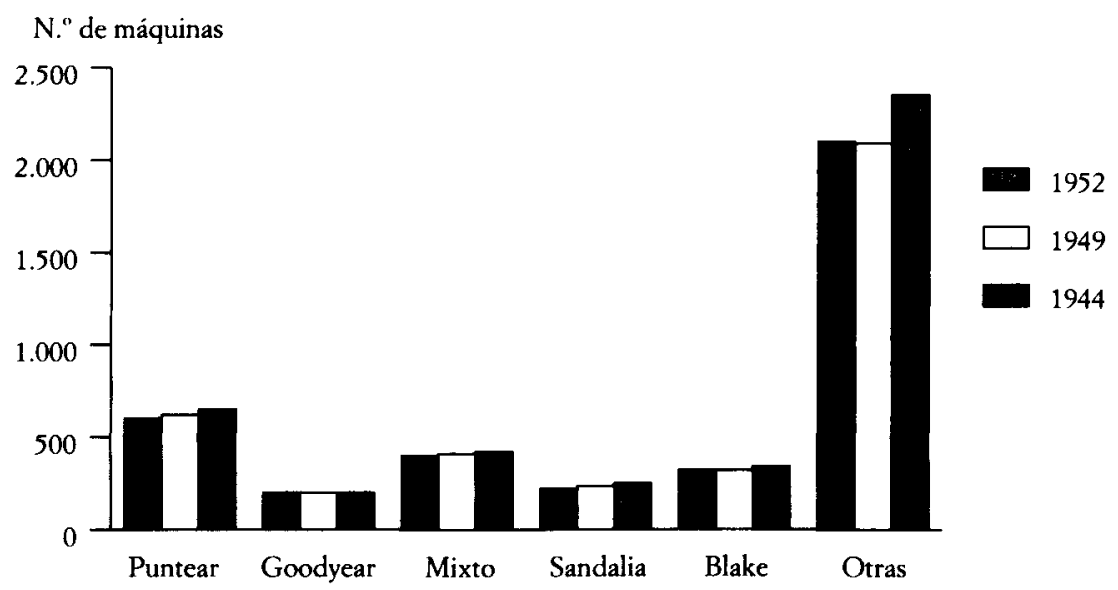

La dependencia tecnológica del exterior en un tiempo de aspiraciones autárquicas provocó que la industria llegase a los años cincuenta con una maquinaria totalmente obsoleta, heredada de los años veinte ${ }^{51}$, y una productividad del trabajo muy alejada de la de otros paises europeos.

Además, el sistema de cupos, especialmente por la falta de agilidad en el comercio intraindustrial que ocasionaba, generó una tendencia al autoabastecimiento en las empresas, que intentaron dotarse del mayor número posible de secciones de fabricación, con el fin de no tener que depender del suministro de semielaborados. En la producción de calzado textil, por ejemplo, las dificultades para proveerse de pisos de caucho llevaron a muchos empresarios a instalar secciones de regenerado de goma y fabricación propia de los pisos; y si no se instalaron más secciones de este tipo en las fábricas fue porque lo impedían los controles administrativos y no era sencillo adquirir la maquinaria 52. La tendencia a autoabastecerse coincidía con la tendencia seguida por algunas

\footnotetext{
5o Piel. Revista española de las industrias de la piel, julio de 1946, p. 4.

"AGA, Sección del Ministerio de Industria, Caja 5.421.

s2 AGA, Sección del Ministerio de Industria, Caja 5.423.
} 
grandes empresas en los años treinta, pero venía a romper con la evolución de la mayoria del sector, que desde finales del siglo XIX habia visto crecer la especialización de las factorias y el fenómeno de la subcontratación.

La intervención generó, pues, dos modelos muy distintos de comportamiento empresarial. Por una parte, la forma de adjudicación de cupos de materias primas y pedidos oficiales, el control sobre importaciones, exportaciones y precios, y la dificultad para obtener prácticamente todos los suministros, alentaron el desarrollo de empresas muy ligadas a la Administración, preparadas para la producción en grandes series y que realizaban por sí mismas buena parte de los semielaborados que el calzado demandaba. De estas empresas, «Silvestre Segarra e Hijos» es sin duda el mejor ejemplo ${ }^{53}$.

Por otro lado, la debilidad de la demanda y la falta de materiales, maquinaria y energía afectaron gravemente a las empresas que antes de la guerra civil habían optado por invertir en la modernización de sus instalaciones. Estas empresas perdieron en la mayoría de los casos su rentabilidad, sin sacar provecho a una maquinaria cuyo mantenimiento resultaba caro y difícil. Podían acceder, en cambio, a la demanda amplia y normalizada de los suministros militares y de los pedidos por parte del Estado para colocar calzado económico en el mercado, y se beneficiaban también de sus mayores cupos de materias primas; pero muchas veces la continuidad de la empresa se debió únicamente a la obtención de los cupos, que se revendian clandestinamente a otras pequeñas fábricas ${ }^{54}$. Estas se adaptaron mucho mejor a la situación: apenas tenian gastos fijos y podían adecuar la producción al débil mercado con un costo mucho menor; necesitaban más mano de obra, pero los salarios eran bajos y, en cualquier caso, quedaba el recurso al clandestinaje, eludiendo los impuestos y las cargas sociales. Además, se organizaron para poder gestionar en común con la Administración los cupos de materiales y el acceso a los pedidos de las instituciones. Así, en el Bajo Vinalopó, donde este tipo de pequeña empresa, basado en las economías de localización, era mayoritario ${ }^{55}$, se crearon con estos fines las sociedades "Fabricantes de Calzado de Elche» en 1942, "Almacenistas de Curtidos Reunidos» en 1945, el «Consorcio de Fabricantes de Tascones» en 1947 y la «Unión de Fabricantes de Calzado de Elche» en 1949.

\footnotetext{
33 Viruela (1988).

54 Bernabé (1976).

35 Miranda (1992).
} 


\section{LA ACTITUD EMPRESARIAL ANTE LA POLITICA ECONOMICA: LA LUCHA CONTRA LA INTERVENCION Y EL PROTAGONISMO DEL SINDICATO VERTICAL DE LA PIEL}

Los grandes empresarios del curtido y el calzado, plenamente identificados con la política económica del régimen, gozaron una situación de privilegio. No hemos encontrado, por ejemplo, ningún testimonio de que a Silvestre Segarra se le negase autorización para alguna importación solicitada o de que no se aceptasen sus propuestas de precios. Este era el tipo de empresas que los politicos de la autarquia tenían como modelo y no dudaron en apoyarlo.

Frente a esta cómoda integración de unos pocos en la economia interveni$\mathrm{da}$, la mayor parte de curtidores y fabricantes de calzado eran conscientes de que la política económica gubernamental les perjudicaba. Su oposición al dirigismo estatal fue canalizada, paradójicamente, a través del Sindicato Vertical de la Piel, que era el encargado en un principio de aplicar las directrices autárquicas en el sector. El Sindicato se convirtió en el organismo representativo de la patronal y, a pesar de gestionar la distribución de cupos y participar en la fijación de las tasas, presionó constantemente para acabar con esta intervención en la industria.

Las solicitudes de liberalización comenzaron después de esos primeros años de posguerra en los que la demanda todavia se mantuvo. En octubre de 1945, los responsables del Sindicato ya solicitaban la desaparición de los precios tasados, la libertad de contratación entre el fabricante y el distribuidor y que los pedidos oficiales se repartiesen entre todas las empresas.

Las primeras medidas liberalizadoras se habían dado en la fabricación de curtidos, a través del sistema de «cupos de reposición por ventas», en 1944. Un año después se pedía la extensión de estas medidas a la industria del calzado, justificada por la estabilización de la demanda. Comenzó así un largo proceso de iniciativas por parte del Sindicato para ir rebajando los controles, proceso en el que chocó con el «ingenierismo» ${ }^{56}$ y la mentalidad ordenancista de ciertos mandos y organismos públicos, singularmente la Comisaria General de Abastecimientos y Transportes (CGAT).

Por fin, después de una entrevista del Jefe Nacional del Sindicato de la Piel, Arcadio Carrasco, con el propio Jefe del Estado en diciembre de 1946, una orden de enero del año siguiente dejó en libertad los precios y la circulación de materias primas. Pero, lógicamente, el cese de las tasas disparó los precios y, en agosto de 1948, los responsables del Ministerio de Industria, que

56 Velasco (1984). 
confiaban más en el autoritarismo que en los mecanismos del mercado, volvieron a restablecer el sistema, traspasando su gestión al Servicio de Carnes, Cueros y Derivados de la CGAT.

El fracaso del Sindicato no fue completo, sin embargo. Mediante asambleas de industriales y comerciantes, y a través de numerosas reuniones con los organismos implicados, se consiguieron algunas concesiones y, en junio de 1952, la libertad de circulación definitiva para los cueros, los curtidos y sus manufacturas.

\section{CONCLUSIONES}

Durante los años cuarenta, las dificultades por las que tuvo que atravesar la industria del calzado, con motivo de la situación heredada de la guerra civil y por la guerra mundial, se vieron agravadas por la política económica que se puso en práctica. Las directrices de esta política, con otras prioridades y por culpa de sus fracasos y de las relaciones laborales autoritarias impuestas, contribuyeron al descenso del poder adquisitivo de los asalariados y, por tanto, al de la demanda de productos de consumo manufacturados. La sobrevaloración de la peseta no facilitó precisamente las exportaciones. La renuncia a los préstamos internacionales y la incapacidad de conseguir divisas se aunaron a las pretensiones autárquicas para limitar las importaciones de materias primas y tecnologia; esta limitación, a su vez, contrajo aún más la oferta de calzado e impidió la renovación del utillaje industrial.

Los precios oficiales de tasa, por debajo de los niveles que habrian alcanzado en una situación de libre mercado, y las asignaciones por parte del Estado de los recursos productivos ocasionaron el desarrollo de un mercado negro de materias primas y el descenso de la calidad de las manufacturas. La imposición de barreras legales para la entrada en la industria o su modernización dificultó esta última y fomentó el clandestinaje. Las restricciones eléctricas frenaron la producción y fomentaron aún más la sustitución de capital por mano de obra.

En definitiva, se consiguió con todo ello que las empresas de calzado españolas tuviesen costes altos y una producción de mala calidad. Cuando, a partir de los años cincuenta, se abrió la posibilidad de acceder a los mercados internacionales, esta desventajosa base retardó el acceso a ellos, limitó los beneficios y condicionó negativamente la posición del calzado español frente a otros competidores. La única baza para la competitividad fueron los bajos salarios. 
Las empresas bien dotadas técnicamente eran la excepción y existía una nube de pequeñas empresas, desarrolladas muchas de ellas en la clandestinidad. Los «monstruos industriales» fomentados por la autarquía, aunque se beneficiaron de la recuperación de los años cincuenta y sesenta, acabaron desplomándose con el final del régimen político que los había alentado, ocasionando graves problemas económicos y sociales alli donde estaban establecidos.

Frente a la incongruencia de la política económica, los empresarios respondieron a través del Sindicato de la Piel, lo que plantea la necesidad de reconsiderar la actuación de estas instituciones verticales en el estudio de la economía española durante el franquismo.

\section{BIBLIOGRAFIA}

BARCIELA, Carlos (1985): «Intervencionismo y crecimiento agrario en España, 19361971», en MARTIN AceÑa, P., y Prados, L. (eds.) (1985), pp. 285-316.

- (1987): «Crecimiento y cambio en la agricultura española desde la Guerra Civil», en Nadal, J.; Carreras, A., y Sudrià, C. (comps.) (1987), pp. 258-279.

Bernabé, José María (1976): La industria del calzado en el Valle del Vinalopó, Valencia, Dto. de Geografia de la Universidad de Valencia.

BuEsa, Mikel (1984): «Las restricciones a la libertad de industria en la política industrial española (1938-1963)», Información Comercial Española, 606, pp. 107-121.

CARRERAS, Albert (1983): La producción industrial española e italiana desde mediados del siglo XIX hasta la actualidad, Tesis doctoral, Universidad Autónoma de Barcelona.

— (1985): «Gasto Nacional Bruto y formación de capital en España, 1849-1979: Primer ensayo de estimación», en Martín Aceña, P., y Prados, L. (eds.) (1985), pp. 17-51.

- (1987): «La industria: atraso y modernización», en NADAL, J.; CarreraS, A., y SUdRIÀ, C. (comps.) (1987), pp. 280-312.

- (1989): «Depresión económica y cambio estructural durante el decenio bélico (19361945)», en Garcfa Delgado, J. L. (ed.) (1989), pp. 3-33.

-(1990): Industrialización española: estudios de historia cuantitativa, Madrid, Espasa Calpe.

Catalan, Jordi (1989): «Autarquía y desarrollo de la industria de fábrica durante la segunda guerra mundial. Un enfoque comparativo", en Garcfa Delgado, J. L. (ed.) (1989), pp. 35-88.

- (1991): «Politica industrial i primer franquisme: l'impacte a Catalunya», L'Avenç. Revista d Història, 149, pp. 28-33.

- (1992): «Reconstrucción, política económica y desarrollo industrial: tres economias del sur de Europa, 1944-1953», en Prados de la Escosura, L., y Zamagni, V. (eds.), El desarrollo económico en la Europa del Sur. España e Italia en perspectiva bistórica, Madrid, Alianza, pp. 359-395.

Clavera, Joan (1976): «Industrialització i canvi de conjuntura en la Catalunya de la postguerram, Recerques, 6, pp. 205-221. 
Clavera, J.; Esteban, J. M.; Mones, M. A.; Montserrat, A., y Ros, J. (1978): Capitalismo español: de la autarquía a la estabilización (1939-1959), Madrid, EDICUSA.

Gaja, E., y Ferrer, M. (1964): 25 años de Paz en Vall de Uxó, Vall de Uxó, Ayuntamiento.

Garcia Delgado, J. L. (1986): «Estancamiento industrial e intervencionismo económico durante el primer franquismo», en FONTANA, J. (ed.), España bajo el franquismo, Barcelona, Crítica, pp. 170-191.

- (1987): «La industrialización y el desarrollo económico de España durante el franquismo», en Nadal, J.; Carreras, A., y Sudrià, C. (comps.) (1987), pp. 164-189.

-ed. (1989): El primer franquismo. España durante la Segunda Guerra Mundial, Madrid, Siglo XXI.

GonzALEZ, Manuel J. (1978): La economía política del franquismo (1940-1970). Dirigismo, mercado y planificación, Madrid, Tecnos.

Martin Aceña, P., y Prados, L. (eds.), La Nueva Historia Económica en España, Madrid, Tecnos.

MELIÁ, Casimir (1955): Industrias químicas y del papel, caucho y cuero en la provincia de Castellón, Castellón, Sociedad castellonense de Cultura.

-(1974): Industrias del vestir (textiles, calzado y diversas) de la provincia de Castellón, Castellón de la Plana, Cámara Oficial de Comercio, Industria y Navegación.

Miranda, José A. (1991): Hacia un modelo industrial. Elche, 1850-1930, Alicante, Instituto de Cultura «Juan Gil-Albert».

- (1992): «Els origens del model industrial valenciä. Elx, 1850-1930», Recerques, 25, pp. 159-173.

Molinero, C. (1991): «Les actituds dels industrials catalans davant la política econòmica del primer franquisme», L'Avenç. Revista d'Història, 149, pp. 54-59.

-e Ysas, P. (1985): «Patria, Justicia y Pan». Nivells de vida i condicions de treball a Catalunya, 1939-1951, Barcelona, La Magrana.

Moreno, Roque (1988): «La escasez de energía eléctrica en la posguerra (1943-1950): su repercusión en la economia alicantina», Anales de la Universidad de Alicante. Historia Contemporánea, 6, pp. 105-126.

Nadal, J.; Carreras, A., y SUdRIÀ, C. (comps.), La economía española en el siglo XX. Una perspectiva bistórica, Barcelona, Ariel.

SANTACreu, José M. (1992): L'economia valenciana durant la guerra civil, Valencia, Institució Valenciana d'Estudis i Investigació.

Sudria, Carles (1987): «Un factor determinante: la energia», en Nadal, J.; Carreras, A. y SUdRiÁ, C. (comps.) (1987), pp. 313-363.

- (1991): «Catalunya i la política economica del primer franquisme. Una reconsideració», L'Avenç. Revista d'Història, 149, pp. 24-27.

Velasco, Carlos (1984): «El ingenierismo como directriz básica de la política económica durante la autarquia (1936-1951)», Información Comercial Española, 606, pp. 97. 106.

Viruela, Rafael (1988): La industria del calzado en la provincia de Castellón, Castellón de la Plana, Sociedad castellonense de Cultura. 